\title{
ESTRUTURA DIAMÉTRICA DA COMUNIDADE E DAS PRINCIPAIS POPULAÇÕES ARBÓREAS DE UM REMANESCENTE DE FLORESTA ATLÂNTICA SUBMONTANA (SILVA JARDIM-RJ, BRASIL) ${ }^{1}$
}

\author{
Fabrício Alvim Carvalho² e Marcelo Trindade Nascimento ${ }^{3}$
}

\begin{abstract}
RESUMO - O objetivo deste trabalho foi avaliar a estrutura diamétrica arbórea de um remanescente de Floresta Atlântica Submontana no Município de Silva Jardim, RJ. Foram utilizadas 20 parcelas de 100 x 5 m, em que todas as árvores com DAP $\geq 5 \mathrm{~cm}$ foram amostradas. A estrutura diamétrica foi analisada em toda a comunidade e nas principais populações (determinadas segundo o VI), a partir de histogramas com intervalos de classes definidos pela fórmula de Spiegel. A análise também considerou os grupos sucessionais das espécies (pioneiras: PI; secundárias iniciais: SI; e secundárias tardias: ST). As curvas (J-reverso) e valores do quociente "q" indicaram ausência de problemas de regeneração das principais populações, a maioria de espécies SI. Entretanto, foi observada tendência de saída de algumas das principais populações de ST (ex. Plathymenia foliolosa e Euterpe edulis), paralelamente à entrada de outras SI. Os resultados complementam as análises florísticas desenvolvidas anteriormente no remanescente, o que indica que a estrutura diamétrica também foi alterada pela fragmentação e pelas perturbações antrópicas pretéritas. Ações coibindo novas perturbações e o enriquecimento através do plantio de mudas das espécies mais afetadas são urgentes, visando à recuperação da qualidade ambiental desse remanescente.
\end{abstract}

Palavras-chave: Estrutura demográfica, grupos ecológicos e sucessão.

\section{DIAMETRIC STRUCTURE OF A TREE COMMUNITY AND ITS MAIN POPULATIONS IN A SUB-MONTANE ATLANTIC FOREST REMNANT (SILVA JARDIM-RJ, BRAZIL)}

\begin{abstract}
This work analyses the tree diametric structure of a Sub-montane Atlantic Forest remnant in Silva Jardim-RJ, Brazil. Twenty $100 \mathrm{~m} \times 5 \mathrm{~m}$ plots were established, and all trees with DBH $\geq 5 \mathrm{~cm}$ were surveyed. The diametric structure was analyzed for the entire community and its main populations (ordered according to the importance value -VI), using diameter class histograms defined by Spiegel's formula. Species were also grouped within successional categories (Pi: pioneer; SI: early secondary; ST: late secondary). The diametric curves ( $J$-reverse shape) and values of Liocourt's ' $q$ ' quotient suggested problems involving lack of regeneration for the main populations for most of the SI species. However, some ST populations (ex. Plathymenia foliolosa and Euterpe edulis) showed a tendency to disappear from the community in the long term, while other SI species became part of the community. These results strengthen the findings of the floristic survey, also suggesting changes in the diametric structure due to the fragmentation process and past disturbance practices. Actions to guarantee protection to the forest remnant and seedling bank enrichment through planting the most threatened tree species are urgently needed for the recovery of the environmental quality of this remnant.
\end{abstract}

Keywords: Demographic structure, ecological groups and succession.

\footnotetext{
${ }^{1}$ Recebido em 28.08.2007 e aceito para publicação em 06.03.2009.

${ }^{2}$ ProgramadePós-GraduaçãoemBiociênciaseBiotecnologiadaUniversidadeEstadualdoNorteFluminense (UENF).E-mail: 〈fabricio.alvim@gmail.com>.

${ }^{3}$ Centro de Biociências e Biotecnologia, Laboratório de Ciências Ambientais da UENF. E-mail: <mtn@uenf.br>.
} 


\section{INTRODUÇÃO}

A organização e manutenção da diversidade podem ser discutidas a partir da análise da estrutura de suas comunidades e populações (CONNELL et al., 1984). As estruturas de populações de plantas resultam da ação de fatores bióticos e abióticos sobre seus indivíduos, sendo as variações espaciais e temporais desses fatores os principais determinantes do arranjo espacial e da estrutura etária das populações e, consequentemente, da comunidade (HARPER, 1990; HUTCHINGS, 1997). No contexto da biodiversidade da Floresta Atlântica, estudos da estrutura de populações e comunidades de plantas são fundamentais para o entendimento dos padrões de distribuição e ocorrência das espécies, assim como para a elaboração de estratégias de manutenção, recuperação e conservação dos remanescentes florestais.

A análise da distribuição diamétrica é importante ferramenta para a avaliação da estrutura florestal. Segundo Gül et al. (2005), a distribuição diamétrica permite examinar as condições dinâmicas da floresta, possibilitando previsões sobre o desenvolvimento das comunidades e populações. Análises de distribuição diamétrica vêm sendo aplicadas em diferentes fitofisionomias do Brasil para compreensão de aspectos relevantes quanto à organização (MARIMON e FELFILI, 2000; SILVA JÚNIOR, 2004; SOUZA et al., 2006; ALVES JÚNIOR et al., 2007), sucessão (PAULA et al., 2004; SIMINSKI et al., 2004) e dinâmica (FELFILI 1997; FERREIRA et al., 1998) do componente arbóreo.

Em florestas tropicais, as comunidades arbóreas apresentam distribuição diamétrica típica na forma exponencial negativa, cuja representação gráfica se assemelha a um J-reverso, em que a maior freqência de indivíduos se encontra nas classes de menores diâmetros (HARPER, 1990). No entanto, perturbações antrópicas podem interferir nessa conformação. Por exemplo, em uma comparação entre uma área explorada e outra não explorada de um remanescente de Floresta Atlântica no Vale do Paraíba do Sul, Minas Gerais, Gomes et al. (2004) mostraram que a exploração madeireira tem levado a alterações significativas na estrutura arbórea, reduzindo a densidade e a área basal de indivíduos das classes diamétricas maiores, levando a um depauperamento do estoque de madeira da floresta explorada e a um comprometimento dos recursos da comunidade florestal. Resultados semelhantes foram encontrados por Villela et al. (2006), que compararam a estrutura arbórea e a dinâmica de nutrientes entre áreas intactas e sujeitas a corte seletivo em uma floresta de Tabuleiro na região Norte Fluminense, incluindo aumento na abundância de espécies secundárias iniciais e decréscimo no estoque de nutrientes da floresta.

Em um estudo recente, Carvalho et al. (2006), analisando a composição e riqueza da flora arbórea de um remanescente de Floresta Atlântica Submontana na região de Imbaú, Silva Jardim, RJ, detectaram alterações em função da fragmentação florestal e perturbações antrópicas pretéritas, como corte seletivo de madeira, queimadas e caça. Seus resultados indicaram predominância de espécies secundárias iniciais, características de florestas perturbadas, na conformação da comunidade arbórea, indicando que o remanescente ainda não alcançou seu desenvolvimento pleno e encontra-se em estágio intermediário de sucessão.

Dando continuidade à avaliação do componente arbóreo desse remanescente, o objetivo deste trabalho foi analisar a estrutura diamétrica de sua comunidade e principais populações. Parte-se da premissa de que a fragmentação florestal da região, aliada às perturbações antrópicas pretéritas, tenham levado a uma alteração também na estrutura diamétrica da comunidade e das principais populações arbóreas, e a uma diferenciação estrutural entre grupos de espécies de estágios iniciais e tardios.

\section{MATERIAL E MÉTODOS}

\section{1. Área de estudo}

A região de Imbaú está localizada no Município de Silva Jardim, RJ (42²8'02'W e 22³7'01'S), e pertence à Área de Proteção Ambiental da Bacia do Rio São João/Mico-Leão-Dourado/IBAMA (PRIMO e VÖLKER, 2003). A região de Imbaú ainda preserva remanescentes de Floresta Atlântica, porém fragmentados e de tamanhos reduzidos, com poucos excedendo 1.000 ha (FUNDAÇÃO SOS MATAATLÂNTICA, 2002), importantes por serem área endêmica de ocorrência do mico-leão-dourado (Leontopithecus rosalia). A vegetação predominante é a Floresta Ombrófila Densa Submontana (VELOSO et al., 1991), em diferentes estágios sucessionais (PRIMO e VÖLKER, 2003). Os solos são classificados como Cambissolos háplicos distróficos (IBGE, 2004). O clima da região está enquadrado pela classificação de Köeppen no tipo As (tropical chuvoso com estação seca no 
inverno). A precipitação anual oscila entre 1.500 e 2.000 $\mathrm{mm}$, sendo os meses de novembro a março os mais chuvosos e de maiores temperaturas (PRIMO e VÖLKER, 2003).

A região de Imbaú formava um maciço florestal contínuo, fragmentado a partir do início do século passado para a implantação de agropecuária extensiva, que resultou na formação de cinco fragmentos florestais de tamanhos variados (19 a 155 ha). Esses fragmentos se encontram isolados há pelo menos 50 anos, situamse muito próximos uns dos outros com distância máxima de cerca de 2 km e estão imersos em uma matriz antrópica composta por pastagens, pequenas culturas de subsistência (frutas cítricas e coco) e áreas de regeneração natural (capoeiras). Embora fragmentada, a vegetação do remanescente mantém similaridade elevada, com valores do índice de similaridade quantitativo (Morisita-Horn) superiores a 50\% entre os fragmentos, conforme descrito por Carvalho et al. (2006).

\subsection{Amostragem da vegetação}

Para a análise da vegetação foi utilizado o método de parcelas longitudinais, visando à obtenção de maior heterogeneidade florística, diminuindo, assim, as chances de a amostragem permanecer restrita a uma mancha vegetal (CAUSTON, 1988). Ao longo de cada um dos cinco fragmentos que compõem o remanescente foram alocadas sistematicamente quatro parcelas de $100 \mathrm{x}$ $5 \mathrm{~m}$ no sentido norte-sul, cada uma distando pelo menos $100 \mathrm{~m}$ da outra e $20 \mathrm{~m}$ das bordas, correspondendo a uma área amostral de 0,2 ha por fragmento e área total amostrada de 1,0 ha.

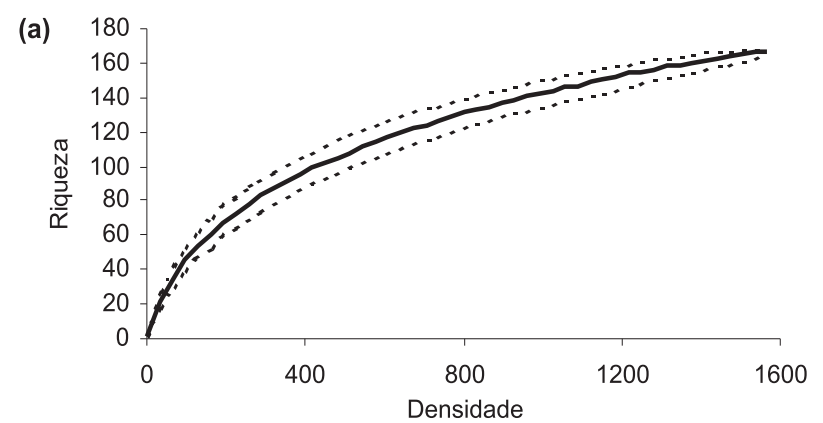

Dentro das parcelas, todas as árvores vivas (com exceção de lianas) com $\mathrm{DAP} \geq 5,0 \mathrm{~cm}$ (diâmetro à altura do peito $=1,30 \mathrm{~m}$ do solo) foram amostradas $\mathrm{e}$ identificadas. A identificação botânica foi realizada no Herbário do Jardim Botânico do Rio de Janeiro (RB), sendo adotada a classificação de famílias de Cronquist (1981). O material coletado encontra-se depositado no Herbário do Centro de Biociências e Biotecnologia da Universidade Estadual do Norte Fluminense (HUENF), e duplicatas foram enviadas para o Herbário RB. Ao todo foram encontrados 1.599 indivíduos pertencentes a 161 espécies, cuja relação completa se encontra em Carvalho et al. (2006). A suficiência amostral foi avaliada a partir de curvas de rarefação (Figura 1), a partir do programa Ecosim (GOTELLI e ENTSMINGER, 2005), e indicou que o esforço amostral foi suficiente para caracterizar a riqueza e diversidade florística da vegetação do remanescente.

\subsection{Análise da estrutura diamétrica}

Para a análise da estrutura diamétrica foram utilizados histogramas de frequência, com intervalos de classes determinados a partir da fórmula de Spiegel (FELFILI e RESENDE, 2003): IC = A/nc, em que Aé a amplitude e nc o número de classes, sendo nc $=1$ $+3,3 \log (\mathrm{n})$, em que $\mathrm{n}$ é o número de indivíduos. $\mathrm{O}$ quociente "q" de Liocourt, que consiste na divisão do número de indivíduos em uma classe de circunferência pelo número de indivíduos na classe anterior, foi utilizado para avaliar o recrutamento (FELFILI e RESENDE, 2003). A relação da distribuição dos indivíduos por classes de diâmetro foi avaliada através de análise de regressão com tendência logarítmica.

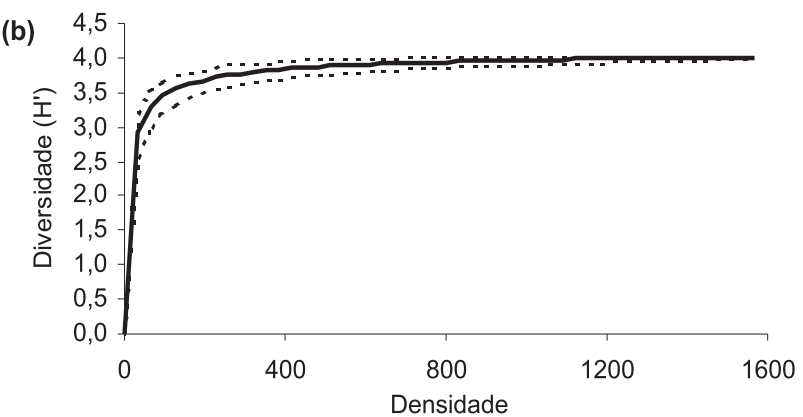

Figura 1 - Curvas de rarefação com valores medianos de riqueza (a) e diversidade (b) (índice de Shannon, H') de espécies arbóreas e seus valores de limite de confiança em 95\% inferiores e superiores (-) no remanescente de Floresta Atlântica Submontana na região de Imbaú, Município de Silva Jardim, RJ.

Figure 1 -Rarefaction curves with median values of the tree species' richness (a) and diversity (b) (Shannon's index), and 95\% upper and lower confidence values (-) for the Sub-montane Atlantic Forest remnant at the Imbaú region, Silva Jardim, RJ, Brazil. 
Essa análise foi realizada tanto para toda a comunidade quanto para as principais populações do remanescente. Assim, foram analisadas as populações das 17 espécies de maior Valor de Importância (VI) na área, pelo fato delas juntas corresponderem a 54\% do VI da comunidade (Tabela 1). A análise também considerou os grupos sucessionais, e para tanto as espécies foram classificadas segundo suas características sucessionais em pioneiras $(\mathrm{Pi})$, secundárias iniciais ( $\mathrm{Si}$ ) e secundárias tardias (St), segundo proposto por Gandolfi et al. (1995). Essa classificação foi baseada em diversos autores, que se utilizaram desses dados nos seus estudos em florestas do domínio da Floresta Atlântica, e aplicada previamente por Carvalho et al. (2006) para a caracterização da vegetação do remanescente em estudo.

\section{RESULTADOS E DISCUSSÃO}

A curva de distribuição dos indivíduos considerando toda a comunidade (Figura 2) seguiu o padrão típico de florestas tropicais inequíneas (HARPER, 1990), ou seja, com distribuição exponencial na forma de J-reverso $\left(\mathrm{R}^{2}=0,70\right)$, em que a maior freqüência de indivíduos se encontrou nas menores classes de diâmetro. Os valores do quociente " $q$ " de Liocourt variaram entre as diferentes classes diamétricas da comunidade (Tabela 2 ), indicando tendências diferenciadas. O valor de "q1" $(0,27)$ esteve abaixo do valor médio de "q" calculado $(0,43)$, evidenciando elevada taxa de recrutamento da comunidade, certamente um reflexo da grande concentração de indivíduos na primeira classe de diâmetro (68\%). Já os valores de “q2" a "q4” foram mais próximos ao valor médio, sugerindo ausência de problemas de regeneração. No entanto, os valores a partir de "q5", que correspondem às maiores classes de diâmetro, afastaram-se do valor médio calculado, sugerindo problemas na manutenção desses estratos.

Tabela 1 - Parâmetros fitossociológicos das espécies arbóreas amostradas no remanescente de Floresta Atlântica Submontana na região de Imbaú, Município de Silva Jardim, RJ; incluídas apenas as 17 espécies ordenadas segundo os maiores valores de importância (VI), Ni: número de indivíduos, DR: densidade relativa, DoR: dominância relativa, FR: frequência relativa, VI: valor de importância e GS: grupo sucessional (Pi: pioneira, Si: secundária inicial e St: secundária tardia)

Table 1 - Phytosociological parameters of tree species sampled in a Sub-montane Atlantic Forest remnant at the Imbaú region, Silva Jardim, RJ, Brazil. Only the 17 most important species were included (ordered by the highest importance value (VI)). Ni: number of individuals; DR: relative density; DoR: relative dominance; FR: relative frequency; VI: importance value; GS: succession group (Pi: pioneer; Si: early secondary; St; late secondary)

\begin{tabular}{|c|c|c|c|c|c|c|c|}
\hline & Espécie & GS & $\mathrm{NI}$ & DR & DoR & FR & $\mathrm{VI}$ \\
\hline 1 & Guarea guidonia (L.) Sleumer & $\mathrm{Si}$ & 220 & 13,75 & 25,81 & 3,27 & 42,83 \\
\hline 2 & Plathymenia foliolosa Benth. & $\mathrm{St}$ & 36 & 2,25 & 15,20 & 2,90 & 20,35 \\
\hline 3 & Cupania oblongifolia Mart. & $\mathrm{Si}$ & 146 & 9,13 & 6,30 & 3,63 & 19,06 \\
\hline 4 & Siparuna guianensis (Tul.) A.DC. & $\mathrm{Si}$ & 75 & 4,69 & 0,77 & 3,09 & 8,54 \\
\hline 5 & Lacistema pubescens Mart. & $\mathrm{St}$ & 64 & 4,00 & 1,05 & 2,72 & 7,77 \\
\hline 6 & Xylopia sericea A. St.-Hil. & $\mathrm{Pi}$ & 47 & 2,94 & 1,95 & 2,36 & 7,25 \\
\hline 7 & Guapira nitida (Mart. ex J.A. Schmidt) Lundell & $\mathrm{Si}$ & 54 & 3,38 & 0,98 & 2,54 & 6,90 \\
\hline 8 & Apuleia leiocarpa Vog. & $\mathrm{Si}$ & 30 & 1,88 & 2,91 & 1,63 & 6,42 \\
\hline 9 & Casearia sylvestris $\mathrm{Sw}$. & $\mathrm{Si}$ & 33 & 2,06 & 1,05 & 2,54 & 5,65 \\
\hline 10 & Nectandra oppositifolia Nees. & $\mathrm{Si}$ & 29 & 1,81 & 0,61 & 2,54 & 4,96 \\
\hline 11 & Brosimum guianense (Aubl.) Huber ex Ducke & $\mathrm{St}$ & 40 & 2,50 & 1,09 & 1,27 & 4,86 \\
\hline 12 & Psychotria velloziana Benth. & $\mathrm{Si}$ & 37 & 2,31 & 0,54 & 2,00 & 4,85 \\
\hline 13 & Nectandra puberula (Schott) Nees & $\mathrm{Si}$ & 26 & 1,63 & 0,83 & 2,36 & 4,82 \\
\hline 14 & Sorocea guilleminiana Gaudich. & St & 37 & 2,31 & 1,75 & 0,73 & 4,79 \\
\hline 15 & Alchornea glandulosa Poepp. & $\mathrm{Pi}$ & 20 & 1,25 & 1,96 & 1,27 & 4,48 \\
\hline 16 & Bathysa mendoncaei K. Schum. & $\mathrm{St}$ & 22 & 1,38 & 1,26 & 1,63 & 4,27 \\
\hline \multirow[t]{3}{*}{17} & Euterpe edulis Mart. & $\mathrm{St}$ & 33 & 2,06 & 0,46 & 1,27 & 3,80 \\
\hline & Total & - & 949 & 59,33 & 64,52 & 37,75 & 161,60 \\
\hline & Demais espécies $(n=144)$ & - & 650 & 40,67 & 35,48 & 62,25 & 138,40 \\
\hline
\end{tabular}

R. Árvore, Viçosa-MG, v.33, n.2, p.327-337, 2009 

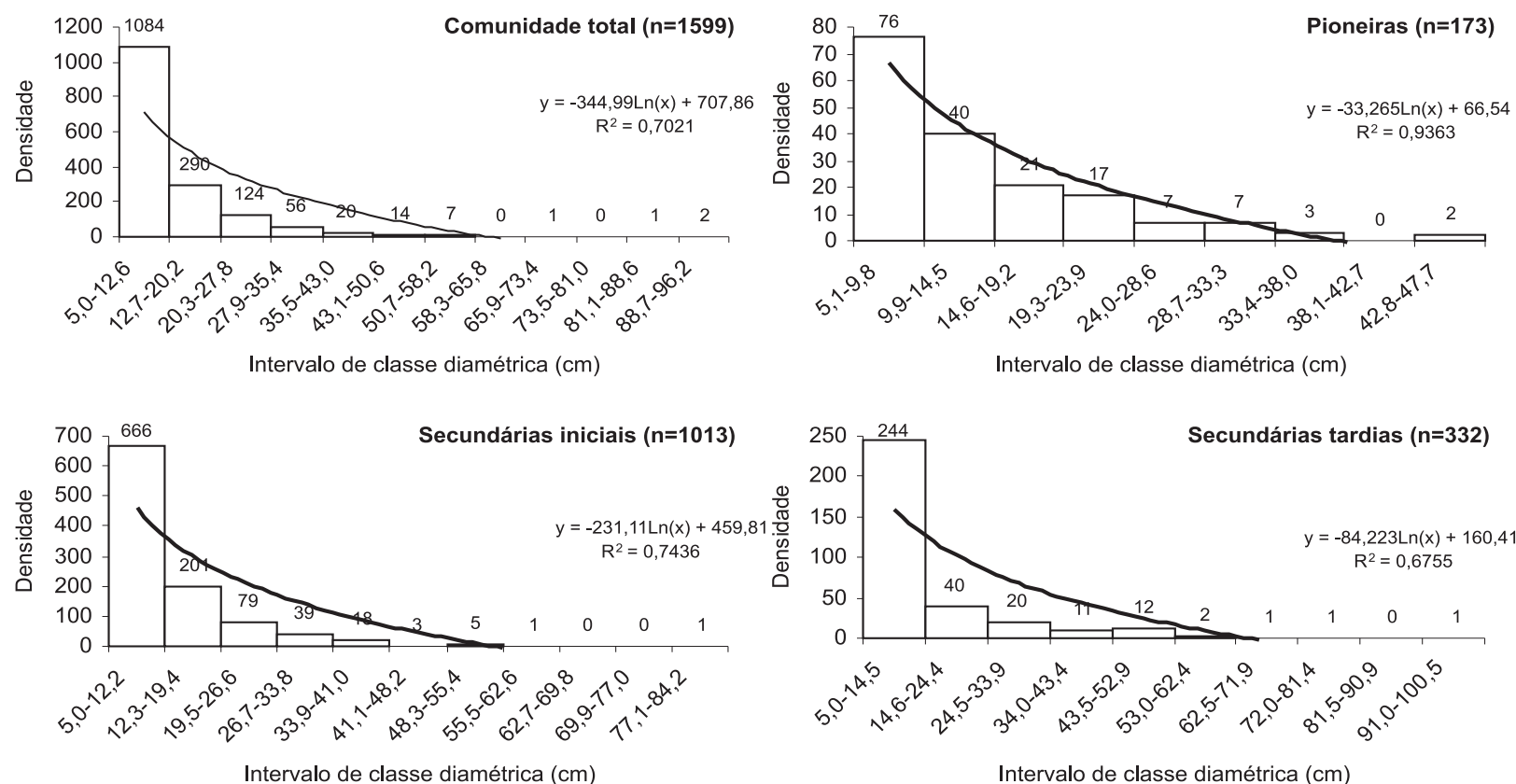

Figura 2 - Distribuição diamétrica dos indivíduos (DAP $\geq 5,0 \mathrm{~cm}$ ) na comunidade e separadamente por classificação sucessional das espécies no remanescente de Floresta Atlântica Submontana na região de Imbaú, Município de Silva Jardim, RJ.

Figure 2 -Diametric distribution of trees $(D B H \geq 5.0 \mathrm{~cm})$ in the Sub-montane Atlantic Forest remnant at the Imbaú region, Silva Jardim, RJ, Brazil. Analysis was carried out of the entire community and successional groups.

Tabela 2 - Valores do quociente “q” de Liocourt da comunidade e populações estudadas, quanto à distribuição diamétrica no remanescente de Floresta Atlântica Submontana na região de Imbaú, Município de Silva Jardim, RJ

Table 2 - Liocourt's “ $q$ " quotient values for the studied community and populations in relation to the diametric distribution in a Sub-montane Atlantic Forest remnant at the Imbaú region, Silva Jardim, RJ, Brazil

\begin{tabular}{lcccccccccccc}
\hline & $\mathrm{q} 1$ & $\mathrm{q} 2$ & $\mathrm{q} 3$ & $\mathrm{q} 4$ & $\mathrm{q} 5$ & $\mathrm{q} 6$ & $\mathrm{q} 7$ & $\mathrm{q} 8$ & $\mathrm{q} 9$ & $\mathrm{q} 10$ & $\mathrm{q} 11$ & $\mathrm{qmédia}$ \\
\hline Comunidade & & & & & & & & & & & & \\
Total & 0,27 & 0,43 & 0,45 & 0,36 & 0,70 & 0,50 & 0,00 & 0,00 & 0,00 & 0,00 & 2,00 & 0,43 \\
Pioneiras & 0,53 & 0,53 & 0,81 & 0,41 & 1,00 & 0,43 & 0,00 & 0,00 & - & - & - & 0,46 \\
Secundárias iniciais & 0,30 & 0,39 & 0,49 & 0,46 & 0,17 & 1,67 & 0,20 & 0,00 & 0,00 & 0,00 & - & 0,37 \\
Secundárias tardias & 0,16 & 0,50 & 0,55 & 1,09 & 0,17 & 0,50 & 1,00 & 0,00 & 0,00 & - & - & 0,44 \\
\hline População & & & & & & & & & & & & \\
Alchornea glandulosa & 0,67 & 0,33 & 0,00 & 0,00 & 0,50 & - & - & - & - & - & - & 0,30 \\
Apuleia leiocarpa & 0,60 & 0,50 & 1,00 & 1,33 & 1,00 & - & - & - & - & - & - & 0,89 \\
Bathysa mendoncaei & 0,17 & 6,00 & 0,83 & 0,20 & 3,00 & - & - & - & - & - & - & 2,04 \\
Brosimum guianense & 0,50 & 1,50 & 0,44 & 1,00 & 1,00 & 0,25 & - & - & - & - & - & 0,78 \\
Casearia sylvestris & 0,55 & 0,83 & 1,00 & 0,20 & 5,00 & - & - & - & - & - & - & 1,52 \\
Cupania oblongifolia & 0,79 & 0,49 & 0,45 & 0,80 & 0,38 & 0,00 & 0,00 & - & - & - & - & 0,42 \\
Euterpe edulis & 1,57 & 0,36 & 0,50 & 0,50 & 0,00 & 0,00 & - & - & - & - & - & 0,49 \\
Guapira nitida & 0,36 & 0,60 & 1,33 & 0,00 & 0,00 & - & - & - & - & - & - & 0,46 \\
Guarea guidonea & 0,58 & 0,52 & 0,34 & 0,27 & 1,67 & 0,00 & 0,00 & 0,00 & - & - & - & 0,42 \\
Lacistema pubescens & 0,48 & 0,79 & 0,36 & 0,75 & 0,33 & 2,00 & - & - & - & - & - & 0,79 \\
Nectandra oppositifolia & 0,12 & 3,00 & 0,00 & 0,00 & 0,00 & - & - & - & - & - & - & 0,62 \\
Nectandra puberula & 0,14 & 0,33 & 0,00 & 0,00 & 0,00 & - & - & - & - & - & - & 0,09 \\
Plathymenia foliolosa & 1,43 & 1,20 & 0,42 & 0,20 & 1,00 & - & - & - & - & - & - & 0,85 \\
Psychotria velloziana & 0,47 & 0,86 & 0,50 & 0,67 & 2,00 & - & - & - & - & - & - & 0,90 \\
Siparuna guianensis & 0,46 & 0,44 & 0,38 & 1,00 & 0,67 & 0,50 & 1,00 & - & - & - & - & 0,64 \\
Sorocea guilleminiana & 0,36 & 1,60 & 0,25 & 2,50 & 0,40 & 0,50 & - & - & - & - & - & 0,94 \\
Xylopia sericea & 0,55 & 0,36 & 0,50 & 3,00 & 0,50 & 0,33 & - & - & - & - & - & 0,87 \\
\hline
\end{tabular}


De fato, essa diferenciação se deve a uma abrupta redução das árvores nas maiores classes de diâmetro, fato relacionado ao corte seletivo pretérito e à mortalidade proporcionada logo após a fragmentação, como observado nos fragmentos recém-isolados na Amazônia Central (LAURANCE et al., 2000). Consequentemente, o aumento no número de indivíduos nas menores classes de diâmetro pode ter sido favorecido pela exclusão dos indivíduos do dossel, facilitando o crescimento de juvenis anteriormente estabelecidos, indicando que nesse remanescente ocorreu alteração nos processos sucessionais internos e na conformação estrutural atual. Esse resultado corrobora com as predições de Parrota (1993), que citou que o aumento na densidade de árvores nas menores classes diamétricas é esperado para florestas secundárias como forma de tamponar os efeitos da perturbação e permitir a continuidade da sucessão.

A análise da distribuição diamétrica da comunidade, considerando separadamente seus três grupos sucessionais (Figura 2), também seguiu o padrão de J-reverso. No grupo das espécies pioneiras foi observada distribuição balanceada por classes de diâmetro $\left(\mathrm{R}^{2}\right.$ $=0,94)$, sugerindo a ausência de problemas de regeneração. Deve-se considerar o fato de que esse grupo corresponde a uma pequena fração do total de indivíduos $(11 \%)$, sendo esperado sua presença em baixa densidade no componente arbóreo, uma vez que são essas as espécies “cicatrizantes" propícias a ocupar áreas alteradas pelas ações antrópicas pretéritas, como nas proximidades das bordas e clareiras no interior do remanescente (BUDOWISK, 1965).

Nos grupos das espécies secundárias iniciais $\left(\mathrm{R}^{2}\right.$ $=0,74)$ e secundárias tardias $\left(R^{2}=0,68\right)$ (Figura 2 ) foi observada elevada concentração de indivíduos na menor classe diamétrica, com valores de "q1" inferiores aos das médias (Tabela 2), indicando elevada regeneração devido à grande entrada de indivíduos e, consequentemente, uma promissora avançada para estágios sucessionais mais avançados. Esses resultados complementam as análises florísticas de Carvalho et al. (2006), no sentido de que evidenciam um franco avanço da comunidade arbórea do remanescente para estágios mais maduros. Isso aparenta estar sendo um reflexo das mudanças no histórico de preservação do remanescente, que há pelo menos uma década se encontra protegido pelos proprietários e, recentemente (2002), foi incluído na Área de Proteção Ambiental da Bacia do Rio São João/Mico Leão Dourado/IBAMA
(FERNANDES et al., 2008), permitindo, assim, que a regeneração natural e os processos sucessionais da vegetação ocorram sem interferências.

Diversos estudos têm sugerindo que a análise da distribuição diamétrica em nível de comunidade, embora importante, pode não ser suficiente para avaliar as condições do componente arbóreo, sendo necessária uma avaliação mais aprofundada, em nível de populações, para complementar a detecção de padrões mais apurados a respeito da estrutura florestal (FELFILI, 1997; MARIMON e FELFILI, 2000; PAULA et al., 2004). Nesse sentido, a análise da distribuição diamétrica das principais populações do remanescente revelou que a grande maioria das populações apresentou distribuição diamétrica balanceada (Figuras 3 a 5; Tabela 2), porém algumas diferenças foram detectadas entre populações de grupos ecológicos distintos.

No grupo das secundárias tardias, as análises revelaram distribuições balanceadas nas três das seis espécies, sendo estas Lacistema pubescens, Brosimum guianensis e Sorocea guilleminiana (Figura 3). No entanto, nas espécies Plathymenia foliolosa, Bathysa mendoncaei e Euterpe edulis foram detectadas distribuições diamétricas desbalanceadas (Figura 3), sugerindo problemas quanto à sua permanência no remanescente.

A espécie Plathymenia foliolosa, a segunda no ranking de VI (Tabela 1), apresentou distribuição diamétrica desbalanceada $\left(\mathrm{R}^{2}=0,36\right)$. Os valores de "q1" $(1,43)$ e "q2" $(1,20)$ estiveram acima do valor médio de "q" calculado $(0,85)$ (Tabela 2$)$, indicando baixa taxa de recrutamento, devido à baixa concentração de indivíduos nas duas primeiras classes de diâmetro (47\%). Isso evidencia que, em longo prazo, a espécie pode não ser capaz de permanecer na comunidade, visto o baixo número de indivíduos nas menores classes diamétricas que irão repor aqueles senis ou em decrepitude. Essa espécie, típica de dossel, é considerada por Lima (2000) como uma das leguminosas mais ameaçadas da Floresta Atlântica do Estado do Rio de Janeiro, devido à drástica redução de suas populações pelo desmatamento e corte seletivo. Portanto, esse resultado foi considerado preocupante, visto que o remanescente estudado é um dos poucos que ainda mantém uma população relativamente grande, mas que aparentemente se encontra comprometida no longo prazo. 

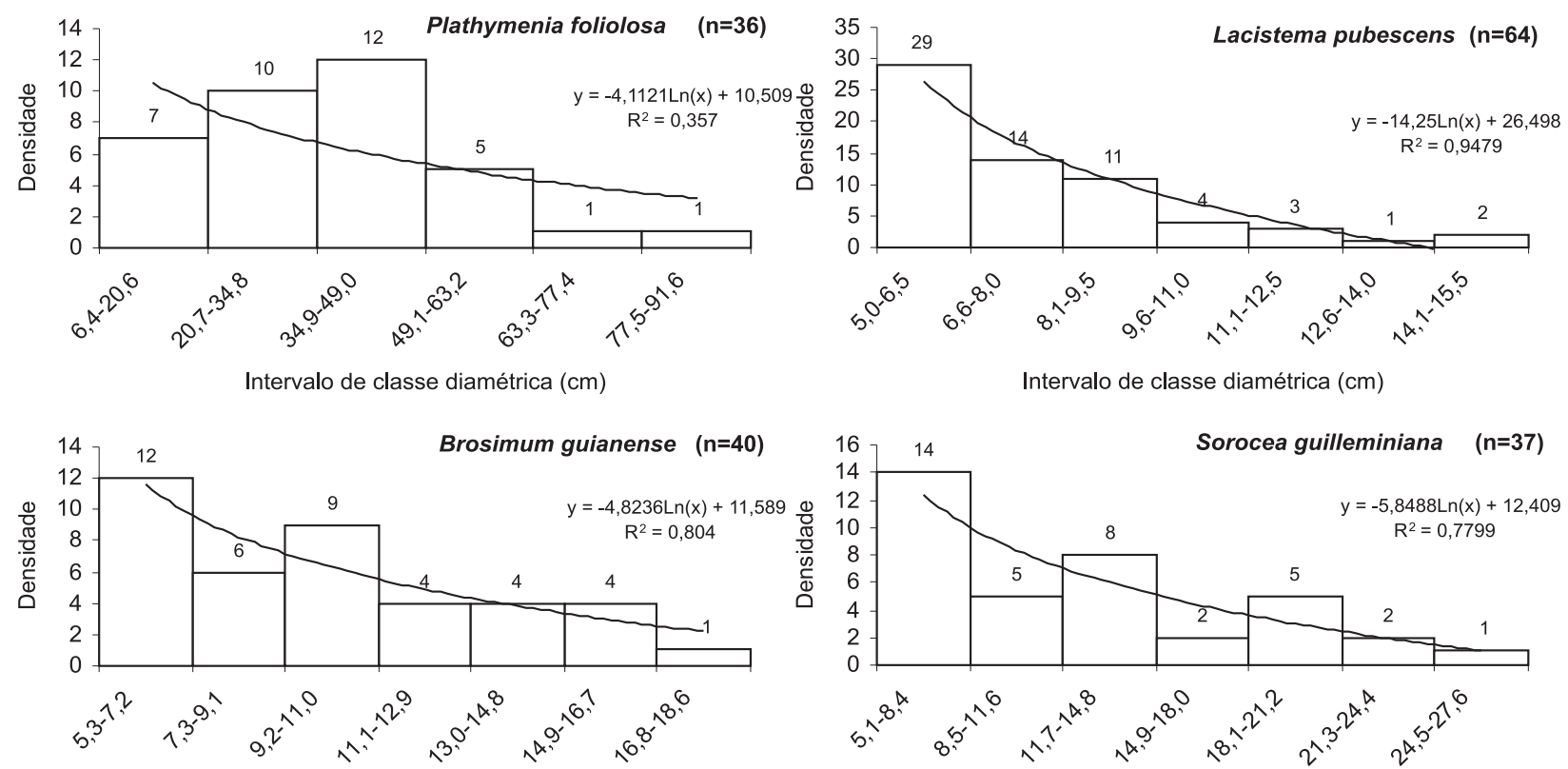

Intervalo de classe diamétrica $(\mathrm{cm})$

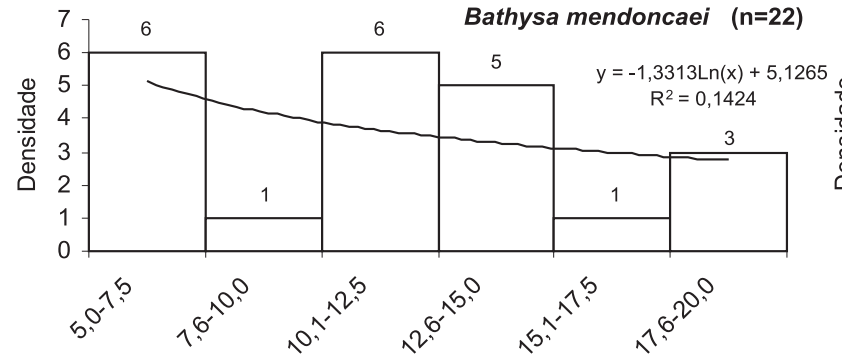

Intervalo de classe diamétrica $(\mathrm{cm})$

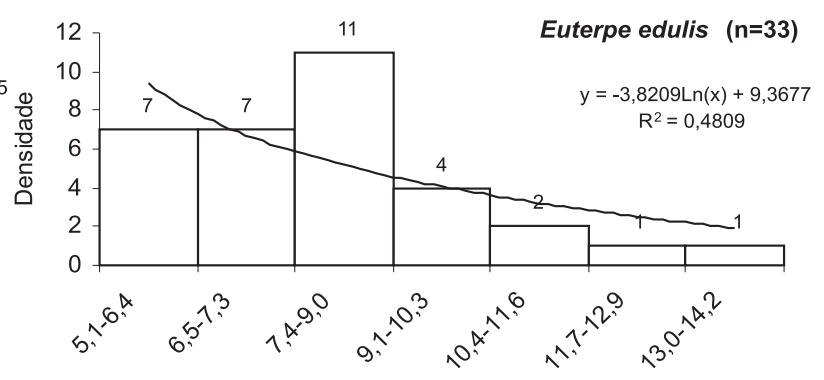

Intervalo de classe diamétrica $(\mathrm{cm})$

Figura 3 - Distribuição diamétrica das espécies secundárias tardias no remanescente de Floresta Atlântica Submontana na região de Imbaú, Município de Silva Jardim, RJ.

Figure 3 - Diametric distribution of the late secondary species in Sub-montane Atlantic Forest remnant at the Imbaú region, Silva Jardim, RJ, Brazil.

Padrão de distribuição diamétrica desbalanceada também foi observado nas espécies Bathysa mendoncaei $\left(\mathrm{R}^{2}=0,14\right)$ e Euterpe edulis $\left(\mathrm{R}^{2}=0,48\right)$, ambas com um baixo número de indivíduos nas primeiras classes diamétricas (Figura 3) e com valores de "q" das primeiras classes muito distintos dos valores médios (Tabela 2). Assim como observado em Plathymenia foliolosa, os resultados evidenciaram que essas espécies poderão apresentar problemas quanto à permanência no remanescente. No caso da espécie Euterpe edulis, outro agravante consiste na sua exploração para produção do palmito, sendo ainda comum encontrar vestígios de corte no remanescente (CARVALHO, 2005). Em síntese, devido ao comprometimento na regeneração, é muito provável que a manutenção dessas populações em longo prazo só seja possível com o término do extrativismo e se realizado um manejo associado ao plantio de mudas, para evitar a perda genética que pode vir a agravar a permanência dessas na comunidade.

Com relação ao grupo das secundárias iniciais (Figura 4) e das pioneiras (Figura 5), as análises revelaram distribuições balanceadas para todas as espécies (Tabela 2), sugerindo ausência de problemas de regeneração e alta capacidade de resiliência dessas populações no remanescente. Entre os principais padrões observados nesse grupo, destacam-se a distribuição balanceada 
e a ausência de problemas de manutenção das espécies de maiores densidades no remanescente (Guarea guidonea e Cupania oblongifolia) (Tabela 1), e o elevado ingresso de indivíduos de espécies características de florestas em estágios sucessionais mais avançados,
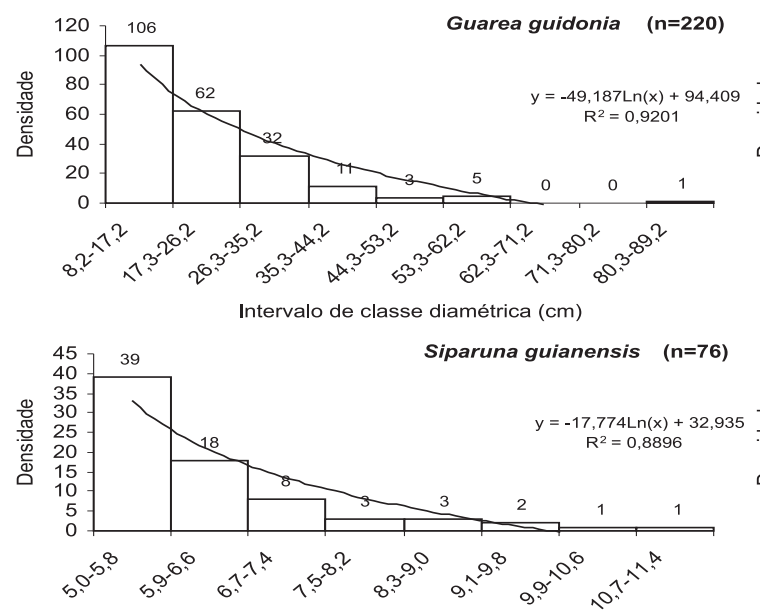

Circunferência $(\mathrm{cm})$

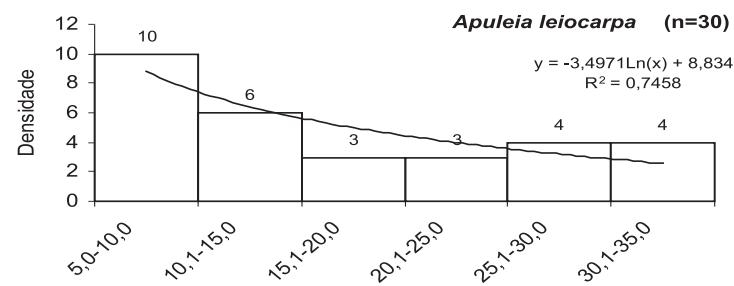

Circunferência $(\mathrm{cm})$
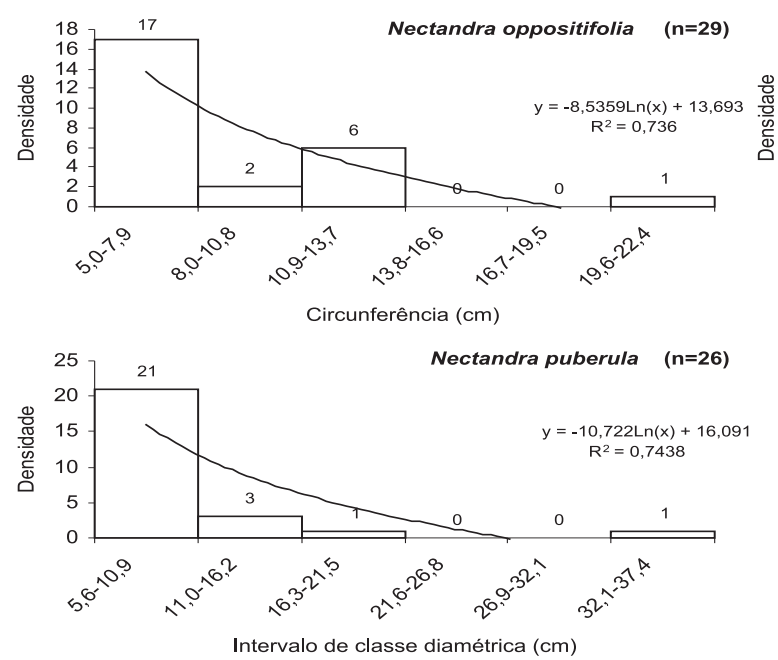

Figura 4 - Distribuição diamétrica das espécies secundárias iniciais no remanescente de Floresta Atlântica Submontana na região de Imbaú, Município de Silva Jardim, RJ.

Figure 4-Diametric distribution of the early secondary species in the Sub-montane Atlantic Forest remnant at the Imbaú region, Silva Jardim, RJ, Brazil.

R. Árvore, Viçosa-MG, v.33, n.2, p.327-337, 2009 como Nectandra oppositifolia e Nectandra puberula (Figura 4), que embora sejam secundárias iniciais, sua presença é comumente registrada em florestas mais maduras (GUEDES-BRUNI, 1998; MORENO et al., 2003; RODRIGUES, 2004).
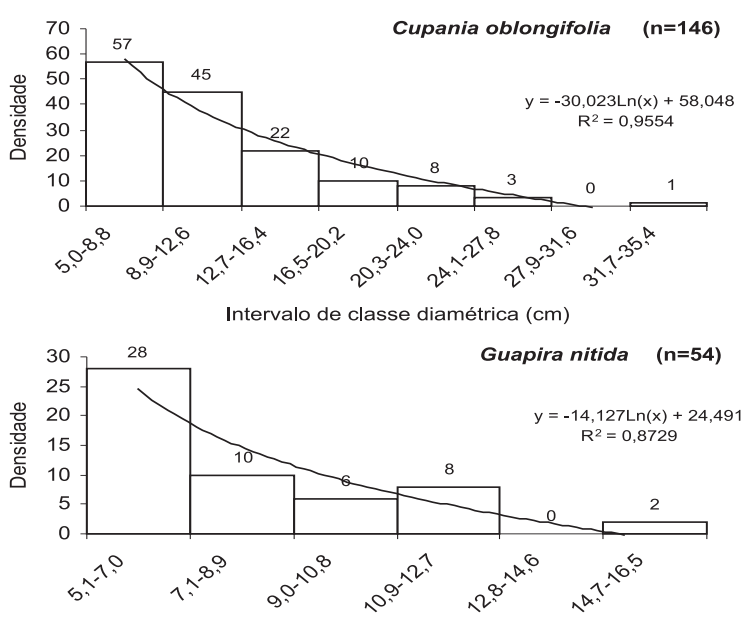

Circunferência $(\mathrm{cm})$
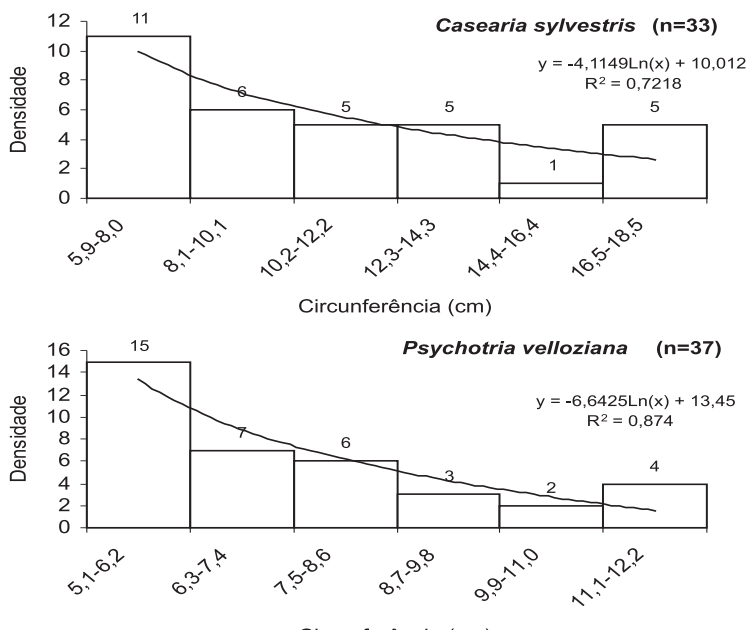

Circunferência $(\mathrm{cm})$<smiles>CCC</smiles>

Circunferência $(\mathrm{cm})$ 


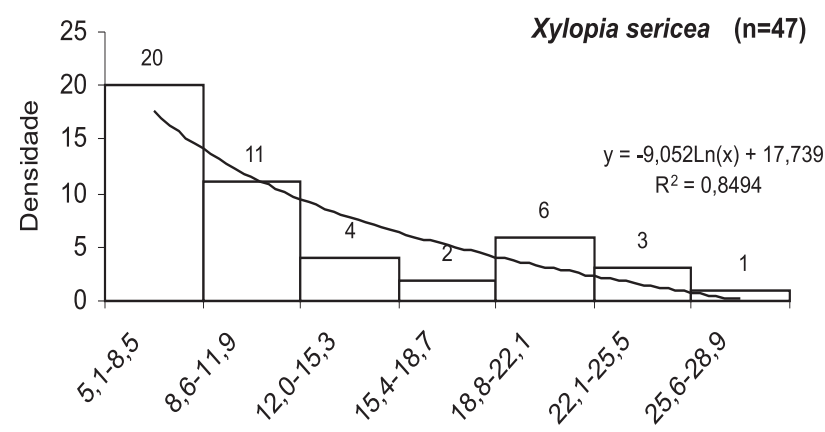

Circunferência (cm)

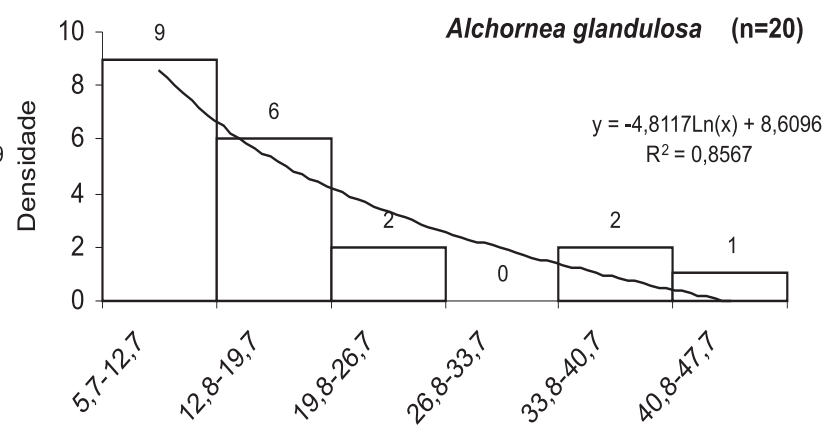

Intervalo de classe diamétrica $(\mathrm{cm})$

Figura 5 - Distribuição diamétrica das espécies pioneiras no remanescente de Floresta Atlântica Submontana na região de Imbaú, Município de Silva Jardim, RJ.

Figure 5 - Diametric distribution of the pioneer species in the Sub-montane Atlantic Forest remnant at the Imbaú region, Silva Jardim, RJ, Brazil.

Em síntese, esses resultados indicam que a comunidade do remanescente tende a manter sua composição estrutural por longo prazo, com ausência de problemas de regeneração de suas principais populações, a maioria das quais secundárias iniciais (ex. Guarea guidonia, Cupania oblongifolia e Siparuna guianensis), entretanto com tendência de saída de algumas das principais populações de secundárias tardias (ex. Plathymenia foliolosa, Euterpe edulis e Bathysa mendoncaei) paralelamente à entrada de outras secundárias iniciais características de estágios sucessionais mais avançados (ex. Nectandra oppositifolia e Nectandra puberula). Esses resultados complementam as análises florísticas anteriormente realizadas e suportam a hipótese de que a fragmentação florestal da região em conjunto com as perturbações antrópicas pretéritas alterou também a estrutura diamétrica da comunidade arbórea, levando a uma diferenciação estrutural entre grupos de espécies de estágios iniciais e tardios. Ações coibindo a ocorrência de novas perturbações como queimadas e o extrativismo de madeira e palmito, associadas ao enriquecimento através do plantio de mudas das espécies mais afetadas (ex. Euterpe edulis, Plathymenia foliolosa e Cariniana legalis, entre outras), são urgentes, visando à recuperação da qualidade ambiental desse remanescente.

\section{AGRADECIMENTOS}

Aos pesquisadores Dorothy S.D. Araujo, Dora M. Villela e Pablo J.F.P. Rodrigues, pela revisão e pelas contribuições à dissertação de mestrado do primeiro autor, que originou este manuscrito; ao técnico Heuzenil S. Cordeiro, pelo auxílio nos trabalhos de campo; aos pesquisadores do JBRJ, especialmente ao João Marcelo A. Braga, pelo auxílio na identificação botânica; ao Alexandro Solorzano, pela revisão do inglês; ao FNMA/ MMA, pelo apoio financeiro; ao LCA/UENF, à Associação Mico-Leão Dourado e ao IBAMA, pelo apoio logístico; à CAPES, pela bolsa de mestrado concedida a Fabrício Alvim Carvalho no período 20032005; e ao CNPq, pela bolsa de produtividade concedida a Marcelo Trindade Nascimento.

\section{REFERÊNCIAS}

ALVES JÚNIOR, F. T. et al. Estrutura diamétrica e hipsométrica do componente arbóreo de um fragmento de Mata Atlântica, Recife-PE. Cerne, v.13, n.1, p.83-95, 2007.

BUDOWISK, G. Distribution of tropical american rain forest species in the light of successional processes. Turrialba, v.15, n.1, p.40-42, 1965.

CARVALHO, F. A.; NASCIMENTO, M. T.; BRAGA, J. M. A. Composição e riqueza florística do componente arbóreo da Floresta Atlântica Submontana na região de Imbaú, município de Silva Jardim, RJ. Acta Botanica Brasilica, v.20, n.3, p.727-740, 2006.

CARVALHO, F.A. Efeitos da fragmentação florestal na florística e estrutura da Mata Atlântica submontana da região de Imbaú, município de Silva Jardim, RJ. 2005. 124f. Dissertação (Mestrado em Biociências e Biotecnologia) - Universidade Estadual do Norte Fluminense, Campos dos Goytacazes, 2005.

R. Árvore, Viçosa-MG, v.33, n.2, p.327-337, 2009 
CAUSTON, D. R. An introduction to vegetation analysis, principles, practice and interpretation. London: Unwin Hyman, 1988. 342p.

CONNELL, J. H.; TRACEY, J. G.; WEBB, L. J. Compensatory recruitment, growth, and mortality as factors maintaining forest tree diversity. Ecological Monographs, v.54, n.2, p.141-164, 1984.

CRONQUIST, A. An integrated system of classification of flowering plants. New York: Columbia University Press, 1981. 555p.

FELFILI, J. M. Diameter and height distribution in a gallery forest community and some of its main species in central Brazil over a six-year period (1985-1991). Revista Brasileira de Botânica, v.20, n.2, p.155-162, 1997.

FELFILI, J. M.; REZENDE, R. P. Conceitos e métodos em fitossociologia. Brasília: Departamento de Engenharia Florestal, Universidade de Brasília, 2003. 64p.

FERNANDES, R. W. et al. Restauração e proteção legal da paisagem - corredores florestais e RPPNs. In: PROCÓPIO de OLIVEIRA, P. P. et al. (Orgs.). Conservação do mico-leão-dourado: enfrentando os desafios de uma paisagem fragmentada. Campos dos Goytacazes: Universidade Estadual do Norte Fluminense, 2008. p.160-179.

FERREIRA, R. L. C.; SOUZA, A. L.; JESUS, R. M. Dinâmica da estrutura de uma floresta secundária de transição: II. distribuição diamétrica. Revista Árvore, v.22, n.3, p.331-344, 1998.

FUNDAÇÃO SOS MATA ATLÂNTICA. Atlas da evolução dos remanescentes florestais e ecossistemas associados no domínio da Mata Atlântica no período 1990-1995. São Paulo: SOS Mata Atlântica/INPE/ISA, 2002.

GANDOLFI, S.; LEITÃO-FILHO, H. F.; BEZERRA, C. L. F. Levantamento florístico e caráter sucessional das espécies arbustivo-arbóreas de uma floresta mesófila semidecídua no município de Guarulhos, SP. Revista Brasileira de Botanica, v.55, n.4, p.753-767, 1995.

R. Árvore, Viçosa-MG, v.33, n.2, p.327-337, 2009
GOMES, A. P. C.; SOUZA, A. L.; MEIRANETO, J. A. Alteração estrutural de uma área florestal explorada convencionalmente na bacia do Paraíba do Sul, Minas Gerais, nos domínios de Floresta Atlântica. Revista Árvore, v.28, n.3, p.407-417, 2004.

GOTELLI, N. J.; ENTSMINGER, G. L. EcoSim: Null models software for ecology, version 7.0. Burlington: Kesey-Bear, Acquired Intelligence, 2005.

GUEDES-BRUNI, R. R. Composição, estrutura e similaridade florística de dossel em seis unidades de Mata Atlântica no Rio de Janeiro. 1998. $231 \mathrm{f}$. Tese (Doutorado em Ecologia) - Universidade de São Paulo, São Paulo, 1998.

GÜL, A. U. et al. Calculation of uneven-aged stand structures with the negative exponential diameter distribution and Sterba's modified competition density rule. Forest Ecology and Management, v.214, p.212-220, 2005.

HARPER, J. L. Population biology of plants. London: Academic Press, 1990.

HUTCHINGS, M. J. The structure of plant populations. In: CRAWLEY, M. J. Plant ecology. Oxford: Blackwell Science, 1997. p.325-358.

\section{INSTITUTO BRASILEIRO DE GEOGRAFIA E} ESTATISTICA - IBGE. Mapa de solos do Brasil. Disponível em: <http:// www.ibge.gov.br $>$ (Acesso em 18 de dez. 2004).

LAURANCE, W. F. et al. Rainforest fragmentation kills big trees. Nature, v.404, p.836, 2000.

LIMA, H. C. Leguminosas arbóreas da Mata Atlântica. 2000. 156f. Tese (Doutorado em Ecologia) - Universidade Federal do Rio de Janeiro, Rio de Janeiro, 2000.

MARIMON, B. S.; FELFILI, J. M. Distribuição de diâmetros e alturas na floresta monodominante de Brosimum rubescens Taub. na Reserva Indígena Areões, Água Boa-MT, Brasil. Revista Árvore, v.24, n.2, p.143-150, 2000.

MORENO, M. R.; NASCIMENTO, M. T.; KURTZ, B. C. Estrutura e composição florística do estrato arbóreo em duas zonas altitudinais na Mata Atlântica de encosta da região do Imbé, RJ. Acta Botanica Brasilica, v.17, n.3, p.371-386, 2003.

R. Árvore, Viçosa-MG, v.33, n.2, p.327-337, 2009 
PARROTA, J. A. Secondary forest regeneration on degraded tropical lands: the role of plantations as faster ecosystems. In: LIETH, H.; LOHMANN, M. (Eds.). Restoration of tropical forest ecosystems. Dordrecht: Kluwer Academic Publishers, 1993. p.63-73.

PAULA, A. et al. Sucessão ecológica da vegetação arbórea em uma floresta estacional semidecidual, Viçosa, MG, Brasil. Acta

Botanica Brasilica, v.18, n.3, p.407-423, 2004.

PRIMO, P. B. S.; VÖLKER, C. M. Bacias

hidrográficas dos Rios São João e das

Ostras - águas, terras e conservação ambiental. Rio de Janeiro: CILSJ, 2003. 115 p.

RODRIGUES, P. J. F. P. A vegetação da Reserva Biológica União e os efeitos de borda na Mata Atlântica

fragmentada. 2004. 136f. Tese (Doutorado em Biociências e Biotecnologia) - Universidade Estadual do Norte Fluminense, Campos dos Goytacazes, 2004.
SILVA JÚNIOR, M. C. Fitossociologia e estrutura diamétrica da mata de galeria do Taquara, na Reserva Ecológica do IBGE, DF. Revista Árvore, v.28, n.3, p.419-428, 2004.

SIMINSKI, A. et al. Sucessão florestal secundária no município de São Pedro de Alcântara, litoral de Santa Catarina: estrutura e diversidade. Ciência Florestal, v.14, n.1, p.21-33, 2004.

SOUZA, D. R. et al. Análise estrutural em floresta ombrófila densa de terra firme não explorada, Amazônia Oriental. Revista Árvore, v.30, n.1, p.75-87, 2006.

VELOSO, H. P.; RANGEL FILHO, A. L. R.; LIMA, J. C. A. Classificação da vegetação brasileira adaptada a um sistema universal. Rio de Janeiro: IBGE, 1991. 124p.

VILLELA, D. M. et al. Effect of selective logging on forest structure and nutrient cycling in a seasonally dry Brazilian Atlantic forest. Journal of Biogeography, v.33, n.3, p.506-516, 2006. 